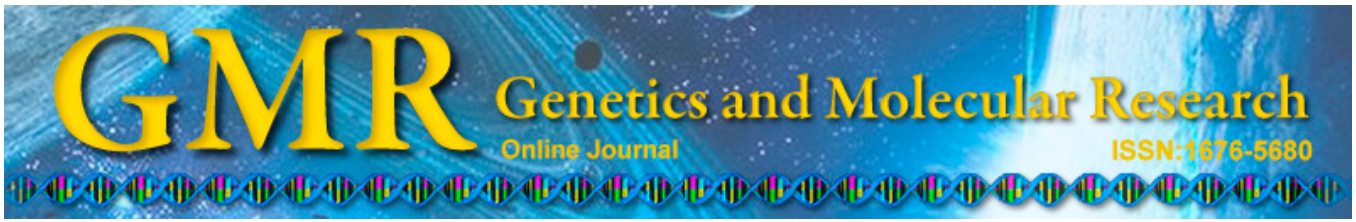

\title{
Investigation of polymorphisms in exon7 of the NSUN7 gene among Chinese Han men with asthenospermia
}

\author{
H.Y. Ren ${ }^{1,2,3}$, R. Zhong ${ }^{1,2,3}$, X.P. Ding ${ }^{1,2,3}$, Z.Y. Chen ${ }^{1,2,3}$ and Y.L. Jing ${ }^{1,2,3}$ \\ ${ }^{1}$ Institute of Medical Genetics, College of Life Science, Sichuan University, \\ Chengdu, China \\ ${ }^{2}$ Bio-Resource Research and Utilization Joint Key Laboratory of Sichuan and \\ Chongqing, Sichuan and Chongqing, China \\ ${ }^{3}$ Key Laboratory of Bio-Resources and Eco-Environment, \\ Ministry of Education, Sichuan University, Chengdu, China \\ Corresponding author: X.P. Ding \\ E-mail: brainding@scu.edu.cn
}

Genet. Mol. Res. 14 (3): 9261-9268 (2015)

Received November 4, 2015

Accepted May 8, 2015

Published August 10, 2015

DOI http://dx.doi.org/10.4238/2015.August.10.6

\begin{abstract}
Previous studies have shown that genetic polymorphisms in exon 7 of the NSUN7 gene can be used as an infertility marker in Iranian men with asthenospermia. However, there have been no equivalent studies in China. In the present study, we investigated the possible association between the genetic polymorphisms in exon7 of NSUN7 and asthenospermia in a Chinese Han population. We recruited 240 asthenospermic men as a patient group and 256 normospermic men as a control group, and analyzed the semen parameters on the basis of World Health Organization (WHO) guidelines. The genetic polymorphisms in exon7 of NSUN7 were detected by DNA sequence analysis. The results were analyzed statistically and a $\mathrm{P}$ value $<0.05$ was considered significant. There were two genetic polymorphisms, c.906C $>\mathrm{T}$ and c.922T $>\mathrm{G}$, in exon7 of NSUN7. We found relatively similar genotypes and allele frequencies between the two groups $(\mathrm{P}=0.928, \mathrm{P}=0.928$,
\end{abstract}


respectively). The combined genotypes of the two polymorphisms did not identify a haplotype associated with asthenospermia $(\mathrm{P}=0.824, \mathrm{P}=$ 0.824 , respectively). Our findings revealed that genetic polymorphisms in exon7 of the NSUN7 gene are not associated with asthenospermia in Chinese Han men.

Key words: Genetic polymorphisms; NSUN7 gene; Asthenospermia

\section{INTRODUCTION}

Parenting is one of the most universally desired goals in adulthood. However, not all people can achieve this desire. Infertility has been recognized as a worldwide health problem. According to incomplete statistics, about $15 \%$ of couples suffer from infertility, which can be attributed to male factors in 50\% of cases (Ferlin et al., 2006). The main symptoms of male infertility are azoospermia, oligospermia, teratospermia, and asthenospermia. Asthenospermia, also known as poor sperm motility, is one of the important factors for male infertility (Aitken et al., 1982a). Sperm cannot travel through a mucus-filled cervix to reach the site of fertilization if the sperm has low motility (Mortimer et al., 1986; Folgerø et al., 1993).

Early studies mainly focused on the effects of mitochondrial genes on asthenospermia, including point mutations and deletions of mitochondrial DNA, which have been reported in patients with low sperm motility (Kao et al., 1995, 1998; Thangaraj et al., 2003; Selvi et al., 2006; Baklouti-Gargouri et al., 2013). In recent years, some autosomal genes, such as TEKTIN (Zuccarello et al., 2008a), DNAII, DNAH5, DNAH11 (Zuccarello et al., 2008b), AKAP3, $A K A P 4$ (Eddy et al., 2003), and SEPT4 (Ihara et al., 2005), have also been confirmed to be correlated with asthenospermia. The NSUN7 gene (NOP1/NOP2/Sun domain family, member 7) encodes an RNA methyltransferase NSUN7, which possess an S-adenosyl methionine binding-domain that may have a role in mitochondrial RNA processing in post-meiotic spermatozoa (Harris et al., 2007). Harris at al. (2007) found that the NSUN7 gene has a role in sperm motility in mice, and revealed a single $\mathrm{C}$ to $\mathrm{T}$ base substitution at nucleotide 1282 in exon7 of NSUN7. In humans, the NSUN7 gene (RefSeq NM_024677.4), which encodes putative methyltransferase NSUN7 with 718 amino acids, is located on chromosome 4 and comprises 12 exons and 11 introns (http://www.ncbi.nlm.nih.gov/gene/79730). Exon7 of NSUN7 is a hot spot and is located in the conserved domains databases (CDD) region (Khosronezhad et al., 2014). A previous study has reported that C26232T (rs2465570) and T26248G (rs2437323) mutations in exon7 of NSUN7 reduced sperm motility in Iranian men (Khosronezhad et al., 2014). However, there have been no equivalent studies in China. The present study aimed to determine whether the genetic polymorphisms in exon7 of the NSUN7 gene are associated with asthenospermia in the Han population of China.

\section{MATERIAL AND METHODS}

\section{Patients and controls}

We recruited 240 asthenospermic men from the Affiliate Hospital of Sichuan Reproductive Health Research Center between September 2013 and July 2014. As a control group, we included 256 men with normal semen parameters who had at least one child. All the semen 
samples were collected and analyzed according to the World Health Organization guidelines (World Health Organization, 1999). The diagnosis of asthenospermia was made on the basis of rapid forward progressive motile sperm (grade A $<25 \%$ ) and total motile sperm (grades A+ $\mathrm{B}<50 \%$ ), with at least one year of infertility. All patients and controls were Han people. The characteristics of the study groups are shown in Table 1.

\begin{tabular}{|c|c|c|}
\hline & Patients $(N=240)$ & Controls $(\mathrm{N}=256)$ \\
\hline Age & $28.6(19-42)$ & $30.1(23-45)$ \\
\hline Ethnicity & Han & Han \\
\hline Motility A (\% motile) & $15.1(3-22)$ & $35.9(29-52)$ \\
\hline Motility $(\mathrm{A}+\mathrm{B})(\%$ motile $)$ & $23.8(4-30)$ & $61.7(54-73)$ \\
\hline No. of live births & 0 & $1.2(1-3)$ \\
\hline
\end{tabular}

All subjects had the normal karyotype, no azoospermia factor microdeletions, and were without unhealthy lifestyles (smoking, drug use, or chemical exposure); they were asked to complete a questionnaire. The subjects gave their informed consent and the study was approved by the local Research Ethics Committee.

\section{Genotyping}

Genomic DNA was extracted from peripheral blood using an Easy Pure Blood Genomic Kit (TransGen, Beijing, China) and stored at $-20^{\circ} \mathrm{C}$ until required for the assay.

The polymerase chain reaction (PCR) primers, 5'AGGTGACAAGGTAATGGGGAA G-3' (forward) and 5'GCAACAAGGACAGCCTGATAAG-3' (reverse), were designed using primer premier 5.0, and synthesized at Sangon Biotech (Shanghai, China). PCR products were processed to a total volume of $25 \mu \mathrm{L}$, containing $100 \mathrm{ng}$ genomic DNA, $2.5 \mu \mathrm{L}$ EasyTag Buffer, $0.2 \mathrm{mM}$ dNTPs, 2 U Taq DNA polymerase (TransGen, Beijing), and $0.3 \mathrm{mM}$ of each primer. The amplification conditions were: denaturing at $95^{\circ} \mathrm{C}$ for 5 min followed by 35 amplification cycles of $30 \mathrm{~s}$ denaturation at $95^{\circ} \mathrm{C}, 30 \mathrm{~s}$ annealing at $59^{\circ} \mathrm{C}, 30 \mathrm{~s}$ extension at $72^{\circ} \mathrm{C}$, and a final extension at $72^{\circ} \mathrm{C}$ for $5 \mathrm{~min}$. The PCR products were sequenced using an ABI 3730xl DNA Analyzer, and sequence analyses were performed using Chromas Pro 2.33 and DNAMAN 8.0.

\section{Statistical analysis}

The genotypes and allelic frequencies between patients and controls were assessed using the chi-squared $\left(\chi^{2}\right)$ test. The odds ratios (ORs) and $95 \%$ confidence intervals $(95 \% \mathrm{CIs})$ were used to estimate the differences in the genotypes and allelic frequencies using a logistic regression model. The statistical analyses described above were performed using the SPSS 19.0 software (SPSS Inc., Chicago, IL, USA). In addition, Hardy-Weinberg equilibrium was determined using the online software SHEsis (http://analysis.bio-x.cn/myAnalysis.php) (Li et al., 2009). The association between the combined genotypes of exon7 of NSUN7 polymorphisms and the risk of asthenospermia were also assessed by the study of haplotypes using Phase 2.1.

All $\mathrm{P}$ values were two-tailed and $\mathrm{P}$ values $<0.05$ were considered to be statistically significant. 


\section{RESULTS}

In our study, we found that two genetic variations in exon7 of the NSUN7 gene, c.906 C $>\mathrm{T}(\mathrm{rs} 2465570)$ and c.922T $>\mathrm{G}(\mathrm{rs} 2437323)$, were present in both the patient and control groups. The results of direct sequencing of exon7 from samples are shown in Figure 1. The results of statistical analysis of the genetic variants in exon7 of the putative NSUN7 gene are summarized in Table 2. Haplotype analysis of the two polymorphisms is shown in Table 3.

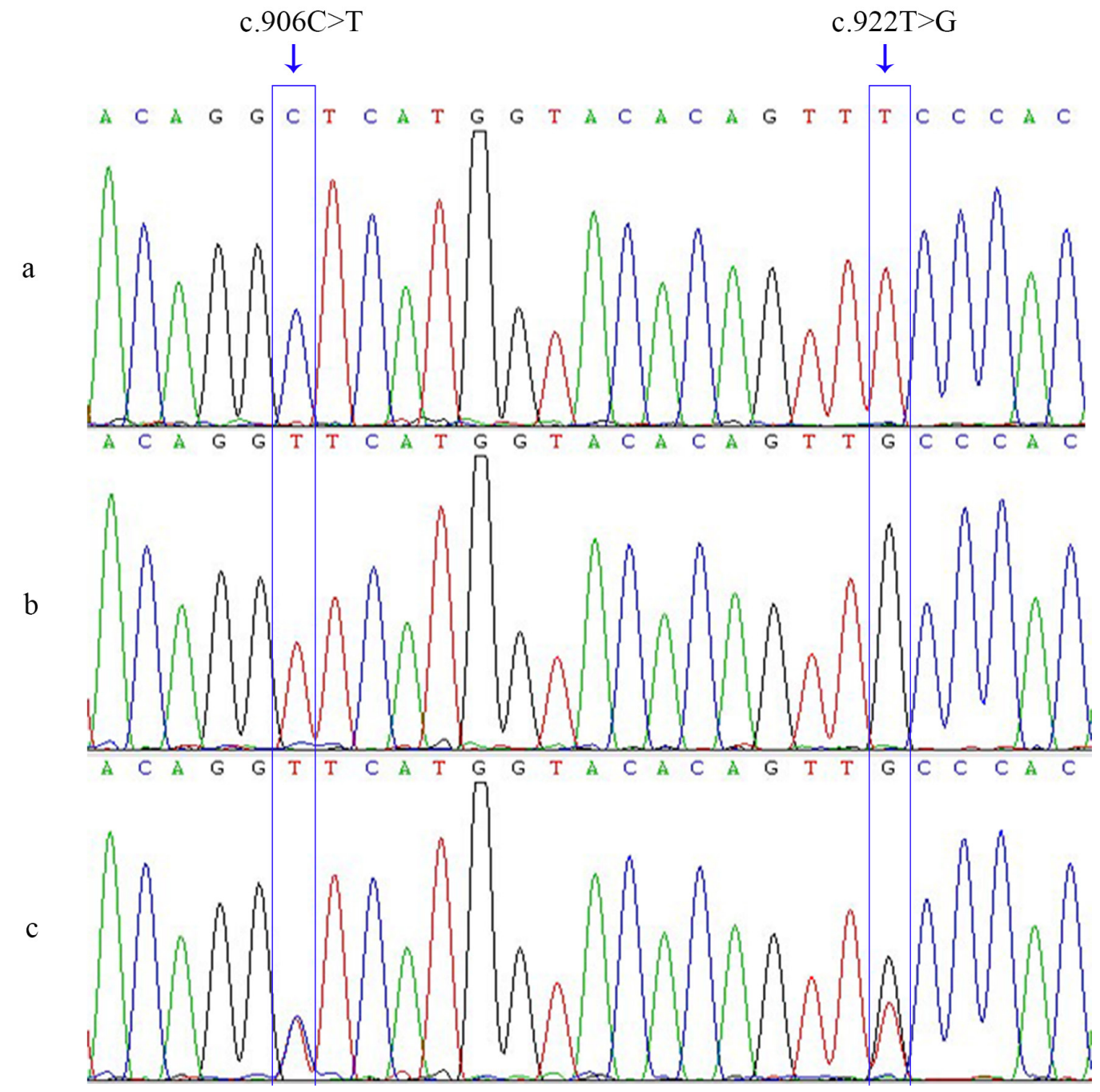

Figure 1. Direct sequencing of exon7 of the NSUN7 gene. (a) CC (c.906C $>$ T) and TT (c.922T $>$ G); (b) TT (c. 906C $>$ T) and GG (c.922T $>\mathrm{G}) ;($ c) $\mathrm{CT}($ c. $906 \mathrm{C}>\mathrm{T})$ and $\mathrm{TG}(\mathrm{c} .922 \mathrm{~T}>\mathrm{G})$. 
Table 2. Genotype and allele frequencies in exon 7 of the $N$ sun 7 polymorphisms among patients and controls.

\begin{tabular}{|c|c|c|c|c|c|c|c|c|c|c|}
\hline \multirow[t]{2}{*}{ Polymorphism } & \multirow[t]{2}{*}{ Subjects } & \multirow[t]{2}{*}{$\mathrm{N}$} & \multicolumn{3}{|c|}{ Genotypes } & \multicolumn{2}{|c|}{ Alleles } & \multirow[t]{2}{*}{ P value } & \multirow[t]{2}{*}{ OR $(95 \% \mathrm{CI})$} & \multirow[t]{2}{*}{ HWE } \\
\hline & & & $\mathrm{N}(\%)$ & $\mathrm{N}(\%)$ & $\mathrm{N}(\%)$ & $\mathrm{N}(\%)$ & $\mathrm{N}(\%)$ & & & \\
\hline $\begin{array}{l}\text { c. } 906 \mathrm{C}>\mathrm{T} \\
\text { rs } 2465570\end{array}$ & $\begin{array}{l}\text { Patients } \\
\text { Controls }\end{array}$ & $\begin{array}{l}240 \\
256\end{array}$ & $\begin{array}{c}\mathrm{CC}(\%) \\
60(25.0) \\
56(21.9)\end{array}$ & $\begin{array}{c}\text { TT (\%) } \\
66(27.5) \\
66(25.8)\end{array}$ & $\begin{array}{c}\text { CT (\%) } \\
114(47.5) \\
134(52.3)\end{array}$ & $\begin{array}{c}\mathrm{C}(\%) \\
234(48.7) \\
246(48.1)\end{array}$ & $\begin{array}{c}\mathrm{T}(\%) \\
246(51.3) \\
266(51.9)\end{array}$ & 0.928 & $1.029(0.723-1.463)$ & $\begin{array}{l}0.444 \\
0.438\end{array}$ \\
\hline $\begin{array}{l}\text { c. } 922 \mathrm{~T}>\mathrm{G} \\
\text { rs } 2437323\end{array}$ & $\begin{array}{l}\text { Patients } \\
\text { Controls }\end{array}$ & $\begin{array}{l}240 \\
256\end{array}$ & $\begin{array}{c}\text { TT (\%) } \\
60(25.0) \\
56(21.9)\end{array}$ & $\begin{array}{l}\text { GG (\%) } \\
66(27.5) \\
66(25.8)\end{array}$ & $\begin{array}{c}\text { TG (\%) } \\
114(47.5) \\
134(52.3)\end{array}$ & $\begin{array}{c}\mathrm{T}(\%) \\
234(48.7) \\
246(48.1)\end{array}$ & $\begin{array}{c}\mathrm{G}(\%) \\
246(51.3) \\
266(51.9)\end{array}$ & 0.928 & $1.029(0.723-1.463)$ & $\begin{array}{l}0.444 \\
0.438\end{array}$ \\
\hline
\end{tabular}

$\mathrm{OR}=$ odds ratio; $\mathrm{CI}=$ confidence interval $\mathrm{HWE}=$ Hardy-Weinberg equilibrium.

Table 3. Haplotype analysis of exon7 of Nsun7 polymorphisms.

\begin{tabular}{lccc}
\hline Haplotype & Patient group frequency (\%) & Control group frequency (\%) & P value \\
\hline C-T & 48.7 & 48.1 & 0.824 \\
T-G & 51.3 & 51.9 & 0.824 \\
\hline
\end{tabular}

Haplotype order is rs2465570 (c.906 C > T) and rs2437323 (c.922T>G).

For the c.906C $>\mathrm{T}$ polymorphism, the frequencies of CC, TT, and CT genotypes were $25,27.5$, and $47.5 \%$, respectively, in the patient group, and $21.9,25.8$, and $52.3 \%$, respectively, in the control group. For the c.922T $>$ G polymorphism, the frequencies of TT, GG, and TG genotypes were $25,27.5$, and $47.5 \%$, respectively, in the patient group, and $21.9,25.8$, and $52.3 \%$, respectively, in the control group.

According to the Hardy-Weinberg principle, the c.906C $>\mathrm{T}$ and c.922 $\mathrm{T}>\mathrm{G}$ polymorphisms were in equilibrium in the patient group $(\mathrm{P}=0.444, \mathrm{P}=0.438$, respectively) and the control group $(\mathrm{P}=0.444, \mathrm{P}=0.438$, respectively).

The allele frequencies of c.906C $>\mathrm{T}$ among patients and controls were 48.7 and $48.1 \%$, respectively, for the $\mathrm{C}$ allele, and 51.3 and $51.9 \%$, respectively, for the $\mathrm{T}$ allele. The allele frequencies of c.922T $>\mathrm{G}$ among patients and controls were 48.7 and $48.1 \%$, respectively, for the $\mathrm{C}$ allele, and 51.3 and $51.9 \%$, respectively, for the $\mathrm{T}$ allele. The statistical analysis revealed no association between c. $906 \mathrm{C}>\mathrm{T}$ and c. $922 \mathrm{~T}>\mathrm{G}$ polymorphisms $(\mathrm{P}=0.928, \mathrm{P}=0.928$, respectively) between the studied groups.

Haplotype analysis of c. $906 \mathrm{C}>\mathrm{T}$ and c.922T $>\mathrm{G}$ polymorphisms showed that there were only two haplotypes (C-T and T-G) in our study, without the C-G and T-T haplotypes, and none of the haplotypes was associated with asthenospermia $(\mathrm{P}=0.824, \mathrm{P}=0.824$, respectively).

\section{DISCUSSION}

Sperm motility is an attribute of semen quality, and is very important for fertilization (Aitken et al., 1982b; Bedford, 1983). Sperm dysfunction has been identified as one of the major factors in male infertility (Hull et al., 1985). Wilson et al. (2005) mutagenized the mouse genome with N-ethyl-N-nitrosourea and found that males homozygous for the Ste5Jcs1 mutation were prone to low sperm motility. Subsequently, Harris et al. (2007) mapped and cloned the Ste5Jcs1 mutation and identified a candidate gene for Ste5Jcs1 called Nsun7, which has a role in sperm motility. The NSUN7 gene encodes an RNA methyltransferase with a Sun do- 
main that is homologous to tRNA and rRNA cytosine methyltransferases. Microarray analysis of the mouse testicular transcriptome indicated that Nsun 7 RNA is abundant in spermatocytes and haploid spermatids (Shima et al., 2004; Harris et al., 2007). The exon 7 of NSUN7 is a hot spot and is located in the CDD region (Khosronezhad et al., 2014). Harris et al. (2007) first found a mutation in the exon7 of Nsun 7 in mice. In humans, Khosronezhad et al. (2014) found two mutations (C26232T and T26248G) in exon7, which can cause deficits in sperm motility in Iranian men with asthenospermia.

We investigated the genetic polymorphisms in exon7 of the NSUN7 gene in the present study and believe we are the first to report c. $906 \mathrm{C}>\mathrm{T}$ and c. $922 \mathrm{~T}>\mathrm{G}$ polymorphisms in Chinese Han people. The polymorphisms we found are the same as in the previous study by Khosronezhad et al. (2014) (c.906C $>\mathrm{T}=\mathrm{C} 26232 \mathrm{~T}$, c.922T $>\mathrm{G}=\mathrm{T} 26248 \mathrm{G})$. The c.906C $>\mathrm{T}$ polymorphism is a silent mutation that converts codon GGC to GGT, both being glycine codons. The c.922 $\mathrm{T}>\mathrm{G}$ polymorphism is a missense mutation that leads to the replacement of serine arising from the TCC codon with alanine from codon GCC.

We evaluated the association of the c.906C $>\mathrm{T}$ and c.922T $>\mathrm{G}$ polymorphisms and their possible association with the risk of asthenospermia. We found relatively similar genotypes and allele frequencies of c. $906 \mathrm{C}>\mathrm{T}$ and c.922 $>\mathrm{G}$ polymorphisms among patients and controls. Haplotype analysis also failed to identify any association with asthenospermia. It is possible that mutations in other genes affect sperm motility in Chinese men. However, Khosronezhad et al. (2014) confirmed that in Iran the two polymorphisms in exon7 of NSUN7 existed only in asthenospermic men but not in the control group.

Prior to this study, only one study has reported the polymorphisms of the NSUN7 gene in humans. Owing to the limited number of relevant studies, the inconsistency in results between our study and the previous study may be attributed to the differences in the studied populations, the sample sizes, and environmental factors. Khosronezhad et al. (2014) studied 100 patients with asthenospermia, but only 30 controls, and the study focused on an Iranian population. In the present study, we included 240 asthenospermic men and 256 controls from a Chinese Han population. Therefore, in the future it would be useful to study a greater variety of ethnicities from different geographical populations comprising large numbers of infertile men with asthenospermia.

Moreover, in the present study we only investigated one exon of the NSUN7 gene; possibly polymorphisms or mutations in other exons of NSUN7 are associated with asthenospermia, so it would be useful to analyze the genetic variants in the whole sequence of the NSUN7 gene in a future study.

In addition, Chi and Delgado-Olguín (2013) studied Nsun7 expression during early embryogenesis in mice in detail and found that $N$ sun 7 was broadly expressed in embryonic and extra embryonic tissues. It is possible that these polymorphisms are related to other diseases. In the future, we will also study the NSUN7 gene and its genetic polymorphisms in relation to other diseases.

Interestingly, although none of the haplotypes was associated with asthenospermia, we found that in two haplotypes (C-T and T-G) the two sites of each haplotype were highly linked, and the two haplotypes had similar frequencies in patients and controls.

In summary, the results of our study showed that c. $906 \mathrm{C}>\mathrm{T}$ and c. $922 \mathrm{~T}>\mathrm{G}$ polymorphisms of exon7 of the NSUN7 gene are not significantly related to asthenospermia in the Chinese Han population. Further studies with larger groups of subjects of different ethnicities 
are required to clarify the association between these polymorphisms and asthenospermia. We plan to investigate all polymorphisms of NSUN7 with regard to their associations with asthenospermia and other diseases, and we will continue to research other factors for asthenospermia in Chinese men.

\section{Conflicts of interest}

The authors declare no conflict of interest.

\section{ACKNOWLEDGMENTS}

We thank the Affiliate Hospital of Sichuan Reproductive Health Research Center for providing us with a platform for the analysis of the semen parameters, and we acknowledge the contributions of the patients and controls who participated in this study.

\section{REFERENCES}

Aitken RJ, Best FS, Richardson DW, Djahanbakhch O, et al. (1982a). The correlates of fertilizing capacity in normal fertile men. Fertil. Steril. 38: 68-76.

Aitken RJ, Best FS, Richardson DW, Djahanbakhch O, et al. (1982b). An analysis of sperm function in cases of unexplained infertility: conventional criteria, movement characteristics, and fertilizing capacity. Fertil. Steril. 38: 212-221.

Baklouti-Gargouri S, Ghorbel M, Ben Mahmoud A, Mkaouar-Rebai E, et al. (2013). Mitochondrial DNA mutations and polymorphisms in asthenospermic infertile men. Mol. Biol. Rep. 40: 4705-4712.

Bedford JM (1983). Significance of the need for sperm capacitation before fertilization in eutherian mammals. Biol. Reprod. 28: 108-120.

Chi L and Delgado-Olguín P (2013). Expression of NOL1/NOP2/sun domain (Nsun) RNA methyltransferase family genes in early mouse embryogenesis. Gene Expr. Patterns 13: 319-327.

Eddy EM, Toshimori K and O’Brien DA (2003). Fibrous sheath of mammalian spermatozoa. Microsc. Res. Tech. 61: 103-115.

Ferlin A, Arredi B and Foresta C (2006). Genetic causes of male infertility. Reprod. Toxicol. 22: 133-141.

Folgerø T, Bertheussen K, Lindal S, Torbergsen T, et al. (1993). Mitochondrial disease and reduced sperm motility. Hum. Reprod. 8: 1863-1868.

Harris T, Marquez B, Suarez S and Schimenti J (2007). Sperm motility defects and infertility in male mice with a mutation in Nsun7, a member of the Sun domain-containing family of putative RNA methyltransferases. Biol. Reprod. 77: 376-382.

Hull MG, Glazener CM, Kelly NJ, Conway DI, et al. (1985). Population study of causes, treatment, and outcome of infertility. Br. Med. J. (Clin. Res. Ed.). 291: 1693-1697.

Ihara M, Kinoshita A, Yamada S, Tanaka H, et al. (2005). Cortical organization by the septin cytoskeleton is essential for structural and mechanical integrity of mammalian spermatozoa. Dev. Cell 8: 343-352.

Kao S, Chao HT and Wei YH (1995). Mitochondrial deoxyribonucleic acid 4977-bp deletion is associated with diminished fertility and motility of human sperm. Biol. Reprod. 52: 729-736.

Kao SH, Chao HT and Wei YH (1998). Multiple deletions of mitochondrial DNA are associated with the decline of motility and fertility of human spermatozoa. Mol. Hum. Reprod. 4: 657-666.

Khosronezhad N, Hosseinzadeh Colagar A and Golam Ali Jorsarayi S (2014). T26248G-transversion mutation in exon7 of the putative methyltransferase NSUN7 gene causes a change in protein folding associated with reduced sperm motility in asthenospermic men. Rep. Fertil. Dev. 27: 471-480.

Li Z, Zhang Z, He Z, Tang W, et al. (2009). A partition-ligation-combination-subdivision EM algorithm for haplotype inference with multiallelic markers: update of the SHEsis (http://analysis.bio-x.cn). Cell Res. 19: 519-523.

Mortimer D, Pandya IJ and Sawers RS (1986). Relationship between human sperm motility characteristics and sperm penetration into human cervical mucus in vitro. J. Reprod. Fertil. 78: 93-102.

Selvi Rani D, Vanniarajan A, Gupta NJ, Chakravarty B, et al. (2006). A novel missense mutation C11994T in the mitochondrial ND4 gene as a cause of low sperm motility in the Indian subcontinent. Fertil. Steril. 86: 1783-1785. 
Shima JE, McLean DJ, McCarrey JR and Griswold MD (2004). The murine testicular transcriptome: characterizing gene expression in the testis during the progression of spermatogenesis. Biol. Reprod. 71: 319-330.

Thangaraj K, Joshi MB, Reddy AG, Rasalkar AA, et al. (2003). Sperm mitochondrial mutations as a cause of low sperm motility. J. Androl. 24: 388-392.

Wilson L, Ching YH, Farias M, Hartford SA, et al. (2005). Random mutagenesis of proximal mouse chromosome 5 uncovers predominantly embryonic lethal mutations. Genome Res. 15: 1095-1105.

World Health Organization (1999). WHO laboratory manual for the examination of human semen and sperm-cervical mucus interaction. 4th edn. Cambridge University Press, Cambridge.

Zuccarello D, Ferlin A, Garolla A, Pati MA, et al. (2008a). A possible association of a human tektin-t gene mutation (A229V) with isolated non-syndromic asthenozoospermia: case report. Hum. Reprod. 23: 996-1001.

Zuccarello D, Ferlin A, Cazzadore C, Pepe A, et al. (2008b). Mutations in dynein genes in patients affected by isolated non-syndromic asthenozoospermia. Hum. Reprod. 23: 1957-1962. 